\title{
INDEX OF SUBJECTS
}

Aberdeen epidemic of milk-borne bacillary dysentery

Agglutination-test for bacillary dysentery

Amino-acids inhibit bacterial growth

Antitoxin, see Diphtheria

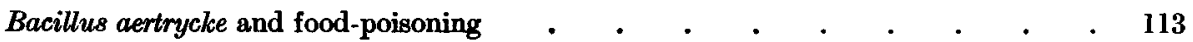

asiaticus, see Parenteric

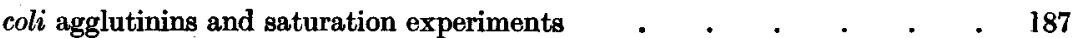

haemolytic and non-haemolytic effect on rabbits . $\quad . \quad$. $\quad . \quad . \quad 180$

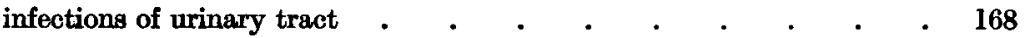

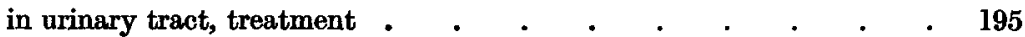

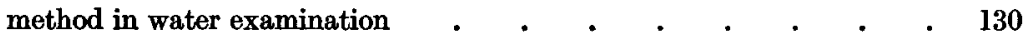

precipitin reactions and complement fixation . . . . . . . 192

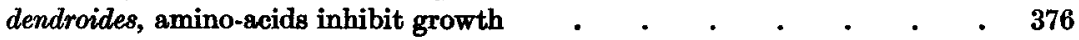

diphtheriae, amino-acids inhibit growth . . . . . . . . . 376

See Diphtheria

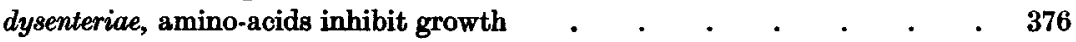

See Dysentery

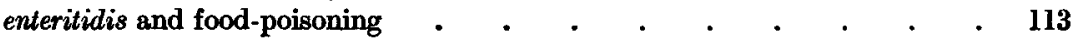

in rats, bearing on food-poisoning $\quad . \quad$. $\quad . \quad . \quad . \quad . \quad 258$

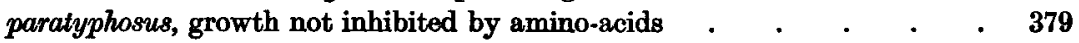

prodigiosus, growth not inhibited by amino-acids $\quad . \quad$. $\quad . \quad$ - $\quad$ - 379

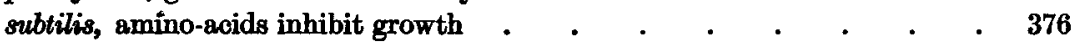

typhosus, growth not inhibited by amino-acids . $\quad . \quad$ • $\quad$. $\quad$. 379

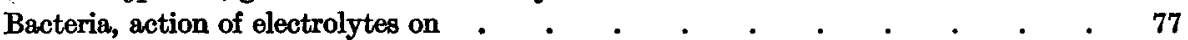

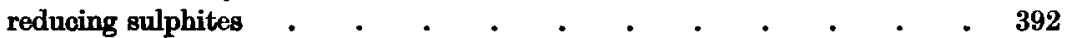

See Diphtheria, Frecal, Haemolytic, Milk, Plague

Bacterial food-poisoning, outbreak in Dublin . . . . . . . . . $\quad 113$

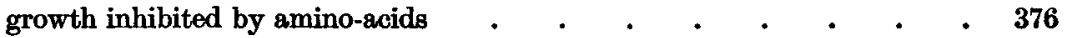

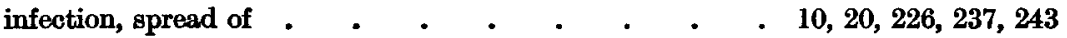

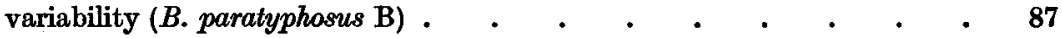

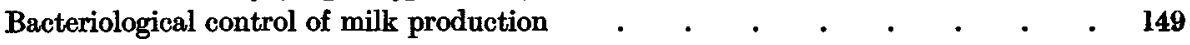
standards, see Milk

Barthel and Jensen's reductase test, see Milk

Blatta, see Cockroaches

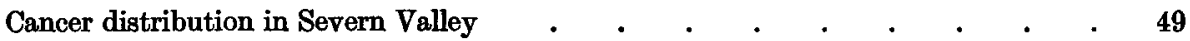

Childhood, see Mortality

Cholera and the ship "Cockroach" . . . . . . . . . . . . . . 359

Cockroaches (Periplanata and Blatta spp.) and cholera . . . . . . . . . 359

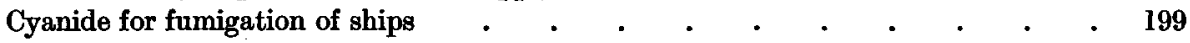

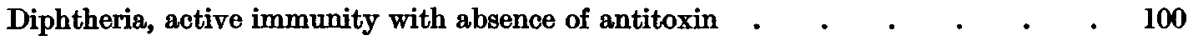

bacilli, virulence tests on $\quad \cdot \quad \cdot \quad \cdot \quad \cdot \quad \cdot \quad \cdot \quad \cdot \quad \cdot \quad, 155$

duration of passive immunity in rabbits $\quad . \quad$. $\quad . \quad$. $\quad . \quad$. 142

Schick dose of toxin as a secondary stimulus . . . . . . 104

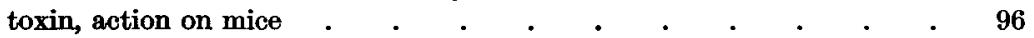

Diphtherial infection in scarlet fever $\quad . \quad$. $\quad . \quad$. $\quad . \quad$. $\quad . \quad$. $\quad 250$ 
Diploma in tuberculous diseases (Cardiff) _. . . . . . . . . $\quad \begin{array}{r}\text { PAGE } \\ 93\end{array}$

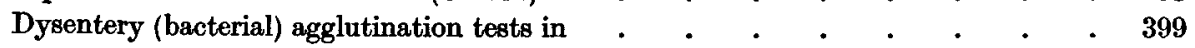

milk-borne epidemic in Aberdeen . . . . . . . 451

Electrolytes, action on bacteria $\quad$. $\quad . \quad$. . . . . . . . . 77

Enteric fever, see Parenteric

Epidemics, experimentally studied in animals . . . . . . 10, 20, 226, 237, 243 - See Plague

Faecal bacteria, see Haemolytic

Food-poisoning outbreaks, bearing of rats on - . . . . . . . 258

See Bacterial

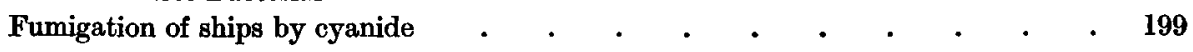

Haemolytic organisms in infants' faeces . . . . . . . . . . . . $\quad$. 37

in intestine . $\quad . \quad . \quad . \quad . \quad . \quad . \quad . \quad . \quad . \quad . \quad 177$

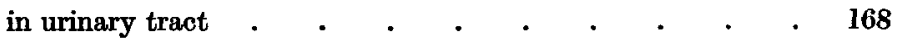

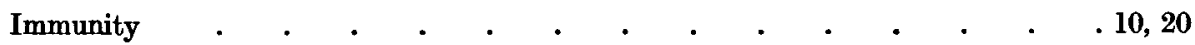

passive, duration of in diphtheria in rabbit $\quad . \quad$. $\quad . \quad . \quad . \quad . \quad 142$

serum constituents, see Wassermann

See Bacterial infection, Neisser

Industrial Hygiene, Section of International Labour Office, Geneva $\quad$. $\quad$ - $\quad$ - 112

Infection, bacterial, spread of . $\quad . \quad$. $\quad . \quad$. $\quad$ - 10, 20, 226, 237, 243

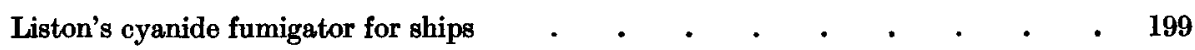

Metabolisms, see Respiratory '

Milk, bacteriological condition and keeping qualities $\quad . \quad$. $\quad . \quad$. $\quad . \quad$. 134

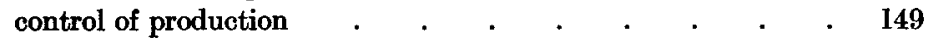

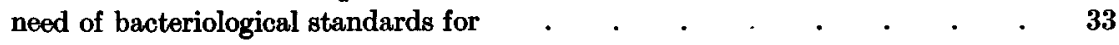

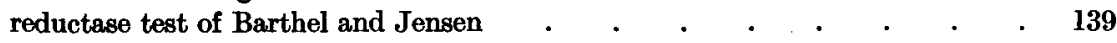

Mortality in childhood with reference to hygiene $\quad$. $\quad . \quad$. . . . . $\quad$. 126

Navy, venereal diseases in $\quad . \quad$. $\quad . \quad$. $\quad . \quad$. . . . . . . 1

Neisser-Wechsberg phenomenon, experimental study of . . . . . . 406

Paratyphoid, see Parenteric

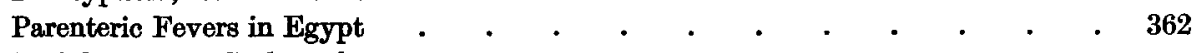

$\begin{array}{ll}\text { Periplanata, see Cockroaches } & \\ & \end{array}$

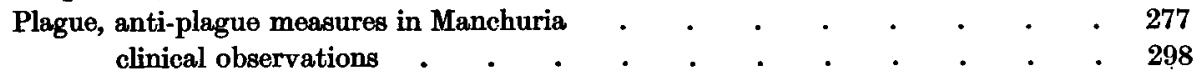

experimental observations

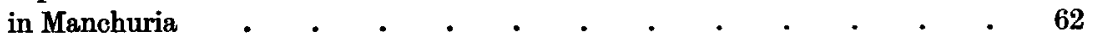

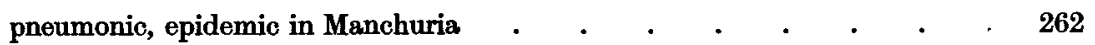

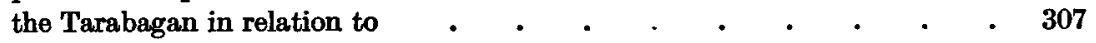

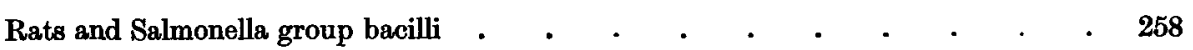

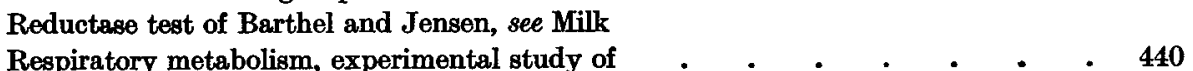

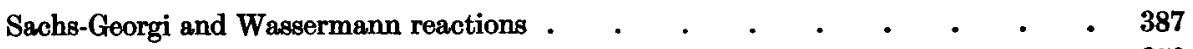

Scarlet fever, diphtherial infection in $\quad \cdot \quad \cdot \quad \cdot \quad \cdot \quad \cdot \quad \cdot \quad \cdot \quad \cdot \quad 250$ 
Schick test, see Diphtheria

Ships, cockroaches and cholera

fumigation by cyanide

Staphylococcus albus and aureus, amino-acids inhibit growth

Toxin, see Diphtheria

Tuberculosis, see Diploma

Typhoid, see Parenteric

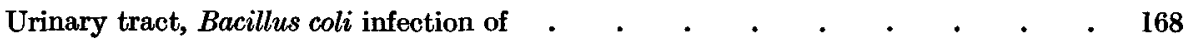

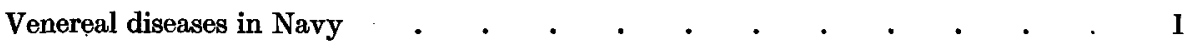

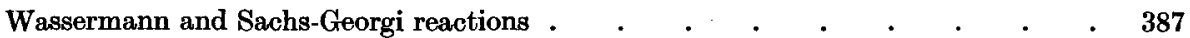

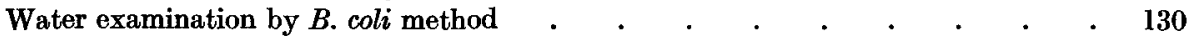

excretally polluted, chemical changes in $\quad . \quad+\quad . \quad . \quad . \quad . \quad . \quad 220$ 